\title{
Genetic associations of Nrf2-encoding NFE2L2 variants with Parkinson s disease a multicenter study
}

Malin von Otter ${ }^{1}$, Petra Bergstrm ${ }^{1 *}$, Aldo Quattrone ${ }^{2,3}$, Elvira Valeria De Marco ${ }^{4}$, Grazia Annesi ${ }^{5}$, Peter Sderkvist ${ }^{6}$, Stephanie Bezzina Wettinger ${ }^{7}$, Marek Drozdzik ${ }^{8}$, Monika Bialecka ${ }^{8}$, Hans Nissbrandt $^{9}$, Christine Klein ${ }^{10}$, Michael Nilsson ${ }^{11,12}$, Ola Hammarsten ${ }^{13}$, Staffan Nilsson ${ }^{14}$ and Henrik Zetterberg ${ }^{1,15}$

\begin{abstract}
Background: The transcription factor Nrf2, encoded by the NFE2L2 gene, is an important regulator of the cellular protection against oxidative stress. Parkinson s disease is a neurodegenerative disease highly associated with oxidative stress. In a previously published study, we reported associations of NFE2L2 haplotypes with risk and age at onset of idiopathic Parkinson s disease in a Swedish discovery material and a Polish replication material. Here, we have extended the replication study and performed meta-analyses including the Polish material and four new independent European patient-control materials. Furthermore, all SNPs included in the haplotype windows were investigated individually for associations with Parkinson s disease in meta-analyses including all six materials.
\end{abstract}

Methods: Totally 1038 patients and 1600 control subjects were studied. Based on previous NFE2L2 haplotype associations with Parkinson s disease, five NFE2L2 tag SNPs were genotyped by allelic discrimination and three functional NFE2L2 promoter SNPs were genotyped by sequencing. The impact of individual SNPs and haplotypes on risk and age at onset of Parkinson s disease were investigated in each material individually and in meta-analyses of the obtained results.

Results: Meta-analyses of NFE2L2 haplotypes showed association of haplotype GAGCAAAA, including the fully functional promoter haplotype $A G C$, with decreased risk $(O R=0.8$ per allele, $p=0.012)$ and delayed onset $(+1.1$ years per allele, $p=0.048)$ of Parkinson $s$ disease. These results support the previously observed protective effect of this haplotype in the first study. Further, meta-analyses of the SNPs included in the haplotypes revealed four NFE2L2 SNPs associated with age at onset of Parkinson s disease (rs7557529 G > A, -1.0 years per allele, $p=0.042$; rs35652124 A > G, -1.1 years per allele, $p=0.045 ;$ rs2886161 $A>G,-1.2$ years per allele, $p=0.021$; rs 1806649 $G>$ $A,+1.2$ years per allele, $p=0.029$ ). One of these ( $r$ 35652124) is a functional SNP located in the NFE2L2 promoter. No individual SNP was associated with risk of Parkinson s disease.

Conclusion: Our results support the hypothesis that variation in the NFE2L2 gene, encoding a central protein in the cellular protection against oxidative stress, may contribute to the pathogenesis of Parkinson s disease. Functional studies are now needed to explore these results further.

Keywords: Parkinson s disease, PD, Nrf2, NFE2L2, Meta-analysis, Multicenter, SNP, Haplotype, Risk factor

\footnotetext{
*Correspondence: petra.bergstrom@clinchem.gu.se

Equal contributors

'Institute of Neuroscience and Physiology, Department of Psychiatry and

Neurochemistry, The Sahlgrenska Academy at the University of Gothenburg,

Bl strket 15, 41345 Gothenburg, Sweden

Full list of author information is available at the end of the article
} 


\section{Background}

Parkinson s disease (PD) is a neurodegenerative disease affecting the central nervous system, resulting in motor symptoms such as rigidity, slowness of movement, postural instability and a characteristic resting tremor. The motor symptoms derive from a decrease in the neurotransmitter dopamine (DA), due to death of dopaminergic (DAergic) neurons in the substantia nigra (SN). Even though several PD-causing genes have been identified, the majority of PD patients have an idiopathic form without known cause and the disease process may involve a combination of genetic and environmental factors (reviewed in [1]). PD pathogenesis is known to involve oxidative stress and the SN seems to be especially vulnerable. Incomplete intracellular oxidation of $\mathrm{DA}$ in the $\mathrm{SN}$, favored by the presence of ferrous iron, may result in the formation of reactive $\mathrm{DA}(\mathrm{semi})$-quinones, which together with decreased levels of reduced glutathione and chronic inflammation adds to the oxidative stress observed in the PD brain (reviewed in [2,3]).

Nuclear factor erythroid 2 (NF-E2) related factor 2 (Nrf2), a transcription factor encoded by the NF-E2-like 2 (NFE2L2) gene, has a key role in the cellular protection against oxidative and electrophilic insults [4]. In the normal state, Nrf2 is kept largely inactive by its repressor protein kelch-like ECH-associated protein 1 (Keap1) [5], which targets Nrf2 for ubiquitin ligation and subsequent degradation by the proteasome [6,7]. When Nrf2 is activated by oxidative or electrophilic stress, it induces transcription of a battery of cytoprotective genes by binding to a specific region in their promoters the antioxidant response element (ARE) $[8,9]$. Nrf2 can also be activated by dietary factors, such as sulforaphane or curcumin, and Nrf2-activating substances have been used in a number of pre-clinical PD models to study the effect of Nrf2 upregulation on PD progression with a pharmacological perspective (reviewed in $[10,11]$. Interestingly, one study has shown that an increased oxidative stress observed in olfactory neurosphere-derived cells from PD patients could be restored by activation of Nrf2 with sulforaphane [12] and curcumin has been shown to protect DAergic SH-SY5Y neurons from 6Hydroxydopamine toxicity [13]. Another study has shown that upregulation of Nrf2 using potent synthetic Nrf2 activators protects DAergic neurons from degeneration in a 1-methyl-4-phenyl-1,2,3,6-tetrahydropyridine (MPTP) mouse model of PD [14].

$\mathrm{Nrf} 2$ is essential in regulation of the cellular redox homeostasis, as it controls rate-limiting steps in the neo-synthesis of glutathione [15], as well as the induction of the antioxidant and neuroprotective enzyme heme oxygenase 1 (HO-1) [16,17]. Another protein regulated by $\mathrm{Nrf} 2$ is the highly inducible $\mathrm{NAD}(\mathrm{P}) \mathrm{H}$ dehydrogenase, quinone 1 (NQO1) [18]. Besides its broad general antioxidant activity, NQO1 prevents toxic redox cycling of DA-quinones through a two-electron reduction into stable hydroquinones [19]. NQO1 has been found to be expressed in the SN of PD patients but not in agematched control subjects [20], suggesting an increased Nrf2 activity in the PD brain. Nrf2 nuclear translocation is enhanced in the PD affected SN [21] and a recent study showing an increased Nrf2 activity in neurons derived from induced pluripotent stem cells (iPSCs) from PD patients [22] indicates a role for Nrf2 activation in the PD pathogenesis. Considering all this, it is feasible that a decreased radical protection due to genetic variation in the NFE2L2 gene could affect the pathogenesis of PD.

We previously reported that a haplotype in the NFE2L2 gene, GAAAA consisting of five tag single nucleotide polymorphisms (SNPs), was associated with delayed age at onset (AAO) in a Swedish discovery material and with decreased risk of PD in an independent Polish replication material [23]. In the same study, investigation of three functional SNPs in the human NFE2L2 promoter, previously shown to influence Nrf2 protein expression $[24,25]$, revealed that the protective haplotype GAAAA was in linkage disequilibrium (LD) with the promoter haplotype AGC, which is part of a fully functional promoter [23].

Here, based on our previous NFE2L2 haplotype associations with PD in the Swedish Gothenburg (PD-Goth) discovery material, seven NFE2L2 haplotypes and one individual SNP were investigated for associations with risk and/or AAO of idiopathic PD in meta-analyses including five independent patient-control replication materials. Furthermore, with an exploratory approach despite lack of association with risk in our previous study, all genotyped SNPs were also independently investigated in metaanalyses including the PD-Goth discovery material.

\section{Methods \\ Patient-control materials}

In total, 2638 individuals (1038 PD patients and 1600 controls) were included in this study. Subjects originated from six independent research centers in Europe: Italy, Malta, Poland and Germany, as well as two independent patient-control sets from Sweden: Gothenburg (PD-Goth) and Linkping (PD-Link).

\section{PD-Goth}

PD-Goth was the discovery material in our previous study [23] and included 165 PD patients and 190 control individuals. All participants were of Caucasian origin and patients and control subjects were of similar age. The patients were diagnosed according to the Parkinson $\mathrm{s}$ Disease Society Brain Bank criteria for idiopathic PD, except that presence of more than one relative with $\mathrm{PD}$ was not considered a criterion for exclusion. PD patients 
with an AAO of $<50$ years were screened to exclude mutations in the recognized PD-causing genes DJ-1, Parkin, PINK1 and LRRK2 $[26,27]$.

\section{Italy}

The Italian material included 329 PD patients and 450 control subjects. All participants were of Caucasian origin and the patients were diagnosed according to the Parkinson s Disease Society Brain Bank criteria for idiopathic PD. The controls were unrelated subjects originating from the same geographical area as the patients and all underwent neurological examination for exclusion of neurological diseases.

\section{PD-Link}

The PD-Link material included 195 PD patients and 379 control subjects collected as part of the Geoparkinson Study [28]. Blood samples were collected from L-dopa positive PD patients in southeast Sweden visiting the Clinic from Geriatrics and Neurology, University Hospital in Linkping, Sweden. The pat ients were classified as having Parkinsons disease or parkinsonism using the United Kingdom Parkinson s Disease Society Brain Bank clinical diagnostic criteria and a neurologist confirmed the diagnosis at recruitment. Individuals with vascular or drug-induced parkinsonism were excluded from the study, as were those with dementia. In this study, only samples from patients with confirmed PD were used. The controls were unrelated Swedish subjects randomly collected from the normal population in southeast Sweden, the same study base as the patients. The patient and control groups were frequency-matched by age and sex.

\section{Malta}

The Malta material included 101 PD patients and 313 control subjects collected as part of the Geoparkinson Study [28]. All participants were of Caucasian (Maltese) origin. The patients were classified as having Parkinsons disease or parkinsonism using the United Kingdom Parkinsons Disease Society Brain Bank clinical diagnostic criteria. In this study, only samples from patients with confirmed PD were used. The controls were from the community or from out-patients at St Lukes Hospital, G Mangia, Malta. They were group-matched to the patients by age and sex.

\section{Poland}

The Polish material was the replication material in our previous study. It included 192 PD patients and 192 control subjects. All participants were of Caucasian origin from the same geographic area, and were matched by sex. Age at sampling (AAS) was significantly higher in the control subjects than in the PD patients (to minimize the risk of PD development in the controls later in life). The patients fulfilled the Parkinsons Disease Society Brain
Bank criteria for idiopathic PD except for the presence of more than one relative with $\mathrm{PD}$, i.e. all patients with a family history of PD were excluded from the study.

\section{Germany}

The German material included 56 PD patients and 76 control subjects. All participants were of Caucasian origin and were matched by age and sex. PD was diagnosed by movement disorders specialists according to the Parkinson s Disease Society Brain Bank criteria for idiopathic PD. The controls were also personally examined and free of any symptoms or signs suggestive of PD.

All materials contained information regarding sex and AAS of patients and control subjects, and AAO of patients (i.e. age at diagnosis (AAD) for Germany, Malta and PD-Link; and age at first symptom (AAFS) for PDGoth, Poland and Italy). Some materials also included information on family history (FH; one or more 1st degree family member(s) with $\mathrm{PD}$ ) and smoking status. Demographics are summarized in Table 1.

\section{SNP selection}

Selection of common SNP genotyping data covering NFE2L2 for the European material CEU (Utah residents with ancestry from Northern and Western Europe) were performed when designing our previous study [23]. In brief, SNP genotyping data was downloaded from the International Haplotype Mapping Project web site (www. hapmap.org) [29] and processed using the Haploview software [30]. LD blocks were constructed according to Gabriel et al. [31] and tag SNPs assigned using the tagger function [30]. A minor allele frequency of $\geq 5 \%$ and pair-wise tagging with a minimum $\mathrm{r}^{2}$ of 0.80 were applied to capture the common SNPs within the block covering the NFE2L2 gene. In the same study promoter SNPs were chosen due to their reported functional effect on Nrf2 transcription [24,25]. The analyzed SNPs, included in the haplotype window previously associated with PD [23], were: five tag SNPs (rs7557529, rs2886161, rs1806649, rs2001350 and rs10183914, here SNPs 2 6) and three functional promoter SNPs [24,25] (rs35652124, rs6706649 and rs6721961, here SNPs P1 P3 [23]), (Figure 1, Table 2).

\section{Tag SNP genotyping and promoter sequencing}

A detailed description of tag SNP genotyping and sequencing of promoter SNPs is provided in our previous study [23]. In brief, all SNPs were genotyped using genomic DNA extracted from blood. All tag SNPs were genotyped using TaqMan Allelic Discrimination [32] with TaqMan Pre-Designed SNP genotyping assays or TaqMan Custom Made SNP genotyping assays (Applied Biosystems, Foster City, CA, USA). The promoter SNPs were genotyped by sequencing, amplifying 
Table 1 Demographic characteristics of PD patients and control subjects

\begin{tabular}{|c|c|c|c|c|c|c|c|c|c|c|}
\hline \multirow[t]{2}{*}{ Parameter } & \multicolumn{3}{|c|}{ Sweden PD-Goth ${ }^{1}$} & \multicolumn{3}{|l|}{ Italy } & & \multicolumn{3}{|c|}{ Sweden PD-Link } \\
\hline & PD & Control & $\mathrm{p}$-value & PD & Control & $\mathrm{p}$-value & & PD & Control & $\mathrm{p}$-value \\
\hline $\mathrm{N}$ & 165 & 190 & & 329 & 450 & & & 195 & 379 & \\
\hline Sex (Male) & $94(57.0)$ & $70(36.8)$ & $<0.001$ & $205(62.3)$ & $179(39.8)$ & $<0.001$ & & $121(62.1)$ & $187(49.3)$ & $0.004^{3}$ \\
\hline AAS (years) & 68.28 .8 & 69.19 .3 & 0.698 & 66.59 .2 & 57.017 .4 & $<0.001$ & 71.4 & 67.5 & $<0.001$ & 3 \\
\hline \multirow[t]{2}{*}{$\mathrm{AAO}^{2}$ (years) } & 59.010 .2 & & & 60.110 .1 & & & & 63.610 .0 & & \\
\hline & $N=164$ & & & $N=328$ & & & & & & \\
\hline \multirow[t]{2}{*}{$\mathrm{FH}$} & $15(9.3)$ & $9(4.8)$ & 0.096 & $24(7.3)$ & ni & ni & & $14(7.2)$ & $15(4.0)$ & 0.095 \\
\hline & $N=162$ & $N=189$ & & & & & & & & \\
\hline \multirow[t]{2}{*}{ Current smoker } & $9(8.7)$ & $13(8.3)$ & 0.897 & $17(8.2)$ & ni & ni & & $9(4.6)$ & $56(14.8)$ & $<0.001$ \\
\hline & & & & $N=208$ & & & & $N=194$ & & \\
\hline \multirow[t]{2}{*}{ Ever smoked } & $38(36.9)$ & $81(51.6)$ & 0.020 & $77(37.0)$ & ni & ni & & $63(32.5)$ & $183(48.3)$ & $<0.001$ \\
\hline & & & & $N=208$ & & & & $N=194$ & & \\
\hline \multirow[t]{2}{*}{ Parameter } & \multicolumn{3}{|l|}{ Malta } & \multicolumn{4}{|l|}{ Poland } & \multicolumn{3}{|l|}{ Germany } \\
\hline & PD & Control & p-value & PD & Control & p-value & & PD & Control & p-value \\
\hline $\mathrm{N}$ & 101 & 313 & & 192 & 192 & & & 56 & 76 & \\
\hline Sex (Male) & $63(62.4)$ & $197(62.9)$ & $0.919^{3}$ & $117(60.9)$ & $117(60.9)$ & $1.000^{3}$ & & $33(58.9)$ & $34(44.7)$ & $0.107^{3}$ \\
\hline AAS (years) & 71.310 .0 & 72.710 .0 & $0.176^{3}$ & 63.710 .9 & 72.99 .9 & $<0.001$ & 4 & 66.011 .9 & 57.810 .8 & $<0.001 \quad 3$ \\
\hline \multirow[t]{2}{*}{$\mathrm{AAO}^{1}$ (years) } & 64.111 .6 & & & 55.210 .9 & & & & 58.712 .5 & & \\
\hline & $N=76$ & & & & & & & $N=54$ & & \\
\hline $\mathrm{FH}$ & $13(12.9)$ & $8(2.6)$ & $<0.001$ & ni & ni & ni & & ni & ni & ni \\
\hline \multirow[t]{2}{*}{ Current smoker } & $3(3.0)$ & $25(8.1)$ & 0.076 & ni & ni & ni & & ni & ni & ni \\
\hline & & $N=308$ & & & & & & & & \\
\hline Ever smoked & $31(30.7)$ & 149 (47.6) & 0.003 & ni & ni & ni & & ni & ni & ni \\
\hline
\end{tabular}

Data are presented as absolute numbers (\%) or mean SD. $\mathrm{p}$-values were calculated with Pearson $\mathrm{X}^{2}$-test for categorical parameters and Mann Whitney $\mathrm{U}$ test for continuous parameters. AAS: age at sampling; AAO: age at onset; AAFS: age at first symptom; AAD: age at diagnosis; ni: no information available. FH: family history (one or more 1st degree family member(s) with PD). N-numbers are shown if data was not available for all subjects in the analysis.

${ }^{1}$ Discovery material on which the replication analyses in this study are based.

${ }^{2}$ AAO is: AAFS for Italy, PD-Goth and Poland; AAD for Germany, Malta and PD-Link.

${ }^{3}$ The results are expected to be similar between the groups due to the applied matching in the original study design. Significant $p$-values are likely a result of not including all samples from the original studies.

${ }^{4}$ This result is expected due to the original study design.

a $423 \mathrm{bp}$ region of the NFE2L2 promoter (forward primer 5-GACCACTCTCCGACCTAAAGG-3, reverse primer 5-CGAGATAAAGAGTTGTTTGCGAA-3, annealing temperature $59 \mathrm{C}$ and 34 cycles on a PTC-200 ThermalCycler (Biorad, Hercules, CA, USA)). Purification of the PCR product was performed with an Illustra GFX PCR Purification Kit (GE Healthcare, Little Chalfont, Buckinghamshire, UK). Sequence reactions were performed using BigDye v3.1 (Applied Biosystems, Forster City, CA, USA) and analyzed on an ABI PRISM 3100 Automated Sequencer (Applied Biosystems, Forster City, CA, USA). Sequence data were analyzed with the DNASTAR SeqMan software (DNASTAR Inc., Madison, WI, USA).

\section{Statistical analyses}

Demographic statistics were performed with SYSTAT11 (SYSTAT Software GmbH, Erkrath, Germany). Pearson
$\mathrm{X}^{2}$-statistics were used for sex, $\mathrm{FH}$ and smoking history; and Mann Whitney U test for AAS.

Genetic association analyses were performed using HelixTree 6.3 (Golden Helix, Bozeman, MT, USA). All tag SNPs were analyzed for deviation from Hardy Weinberg equilibrium. Based on the significant results in the PD-Goth material [23], haplotypes were analyzed for associations with PD (GAAAA, GAGGG and GAAAG in the haplotype window consisting of tag SNPs 26 , AGC in the window consisting of promoter SNPs 13 and GAGCAAAA, GAGAAGGG and GAGCAAAG in the window consisting of all eight SNPs combined, see Figure 1 and Table 2 for SNP overview). A detailed description of how the haplotype windows were identified is given in our previous study [23], where the identified windows were corrected using permutations tests with 10000 permutations and $\mathrm{p}_{\mathrm{c}}$-values of $\leq 0.05$ were considered statistically significant. 


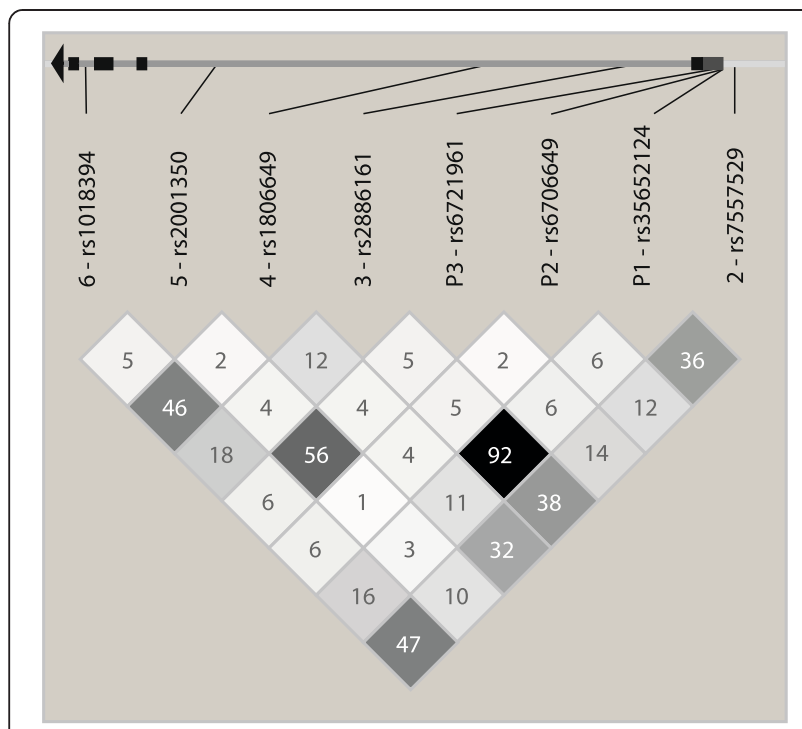

Figure 1 Schematic NFE2L2 gene positions of the studied SNPs and their LD-patterns, showing $\mathrm{r}^{2}$-values as $100 \mathrm{r}^{2}$.

With meta-analysis sample sizes $>2000$ individuals (after correction for unequal sample sizes) and a desired power of $80 \%$, we can detect standardized differences down to approximately 0.13 in this study [33], which means that all the detected significances in risk and AAO reported in our previous paper [23] can be detected here at the applied significance level of 0.05 .

SNP and haplotype associations were analyzed using logistic or linear haplotype regression, where each haplotype was analyzed individually relative to all the other haplotypes together. Sex was the only covariate available in all materials. In order to maximize sample size and power of the analyses and to make the results comparable between the independent materials, only sex was included as covariate in our model (except for risk of PD in the Polish material where the number of males and females were identical in the patient and control groups). However, when data was available and statistically relevant (see our previous study [23] for a detailed description of how statistically relevant covariates were selected), the effects of sex, FH and smoking habits were evaluated in the meta-analyses to assure that exclusion of covariates did not affect the outcomes. Haplotype frequencies were estimated in each material individually using the EM algorithm [34] allowing imputation to compensate for missing genotypes.

Meta-analyses were performed using inverse-variance weighting. In attempts to replicate our previously observed associations, the five replication materials were analyzed for associations of NFE2L2 with PD risk and AAO. One-sided p-values $\leq 0.05$ were considered statistically significant. In the exploratory meta-analyses for individual SNP associations with PD risk and AAO, all six materials were included in the analyses. Two-sided p-values $\leq 0.05$ were considered statistically significant.

\section{Ethics}

The study was approved by ethics committees in each respective country (Sweden Gothenburg: the regional ethics committee at University of Gothenburg, Sweden; Italy: the ethical committee at the University Hospital Mater Domini, Catanzaro, Italy; Sweden Linkping: the regional ethics committee at University of Linkping, Sweden; Malta: the research ethics committee at the University of Malta, Malta; Poland: the ethics committee of the Pomeranian Medical University, Szczecin, Poland; Germany: the ethics committee of the University of Lbeck). This study was in compliance with the Helsinki Declaration of 1975 and written informed consent was obtained from all participants.

Table 2 Overview of the studied SNPs

\begin{tabular}{|c|c|c|c|c|c|c|}
\hline SNP & rs-ID & Genome position & Alleles $d>D$ & Gene location & SNP type & Taqman assay \\
\hline NFE2L2 & & Chr:2(-) & & & & \\
\hline 2 & rs7557529 & 177843343 & $G>A$ & 5'-region & & C__436313_10 \\
\hline P1 & rs35652124 & 177838319 & $A>G$ & Promoter $(-653)$ & Regulatory $^{1}$ & Sequencing ${ }^{2}$ \\
\hline P2 & rs6706649 & 177838317 & $G>A$ & Promoter (-651) & Regulatory $^{1}$ & Sequencing ${ }^{2}$ \\
\hline P3 & rs6721961 & 177838283 & $C>A$ & Promoter $(-617)$ & Regulatory $^{1}$ & Sequencing ${ }^{2}$ \\
\hline 3 & rs2886161 & 177836085 & $A>G$ & Intron 1 & & C__351881_10 \\
\hline 4 & rs1806649 & 177826398 & $G>A$ & Intron 1 & & C_11634983_10 \\
\hline 5 & rs2001350 & 177808671 & $A>G$ & Intron 1 & & C_11634985_10 \\
\hline 6 & rs10183914 & 177805912 & $G>A$ & Intron 3 & & C_157561_10 \\
\hline
\end{tabular}

The SNPs analyzed in the study are numbered according to gene location in reference [23]; SNPs 2 are tag SNPs; SNPs P1 P3 are functional promoter SNPs. Genome positions were obtained from the CEU population of the HapMap Genome Browser (Phase $1 \& 2$ full dataset). Alleles are given according to the sense sequence of the gene.

${ }^{1}$ See reference [25].

${ }^{2}$ See reference [23]. 


\section{Results}

\section{Demographics}

PD-Goth: Patients and control subjects were similar in age, FH and current smoking status, but differed significantly in the distributions of sex and ever-smoker status. Italy: Patients and control subjects differed significantly in AAS and sex. No information was available regarding FH or smoking habits for the control subjects. PD-Link: Patients and control subjects did not differ with respect to $\mathrm{FH}$, but differed significantly in AAS, sex and smoking status. Malta: Patients and control subjects were similar in AAS, sex and current-smoker status, but differed significantly with respect to $\mathrm{FH}$ and ever-smoker status. Poland: Patients and control subjects were matched in sex, but differed significantly in AAS due to the study design. No information was available regarding PD FH or smoking habits for either PD patients or control subjects. Germany: Patients and control subjects were similar regarding sex frequencies, but differed significantly in AAS. No information was available regarding PD FH or smoking habits for either PD patients or control subjects.

In general, all centers had a higher percentage of males relative to females amongst the patients (57.0 62.4\%). Amongst the controls there was a wider range (36.8 $62.9 \%$ ) due to different study designs. Similarly, the average AAS was more similar between the patient groups (63.7 71.4 years) than between the control groups (57.0 72.9 years). The Polish material stood out, since the controls were older than the patients as a result of the study design. The average AAO varied between 55.2 and 64.1 years and the average time from AAO until AAS ranged from 6.4 to 9.2 years between the different patient groups. Where data was provided, FH of PD was always more common in the patient groups than in the controls, though this difference did not always reach statistical significance. As expected, smoking (ever smokers) were more common in all patient groups than in the control groups, whenever data was available. All demographics are given in Table 1. Frequencies for the studied NFE2L2 SNPs and haplotypes in patients and controls are summarized in Additional file 1: Table S1 and Additional file 2: Table S2, respectively.

\section{Tag SNP genotyping}

None of the studied SNPs had a Bonferroni-corrected Hardy-Weinberg equilibrium p-value of $<0.001$. The overall call rate was $>95 \%$.

\section{Haplotype associations}

In accordance with the findings in the first study, metaanalyses of NFE2L2 haplotypes including five independent replication patient-control materials showed a protective effect of the two haplotypes GAGCAAAA and GAAAA on PD. Haplotype GAGCAAAA, consisting of the five tag SNPs and the three functional promoter SNPs (SNPs 2, P1, P2, P3, 3, 4,5 and 6 according to gene direction, for rs numbers see Table 2), was associated with both decreased risk $(\mathrm{OR}=0.8$ per allele, $\mathrm{p}=0.012$ ) (Table 3 , Figure $2 \mathrm{~A}$ ) and later $\mathrm{PD}$ onset $(+1.1$ years per allele, $\mathrm{p}=0.048)$ (Table 4, Figure 2B). Haplotype GAAAA, consisting of tag SNPs 26 only, was associated with later PD onset $(+1.3$ years per allele, $\mathrm{p}=0.024)$ (Table 4$)$ and showed a tendency towards association with decreased PD risk $(\mathrm{OR}=0.9$ per allele, $\mathrm{p}=0.052)$ (Table 3$)$.

None of the haplotypes GAGAAGGG, GAGGG, GAGCAAAG or GAAAG in the same two windows, all associated with increased risk in the PD-Goth material in the first study, was significantly associated with PD in the meta-analyses. The promoter haplotype AGC alone was not associated with PD in meta-analysis (Tables 3 and 4).

\section{Individual SNP associations}

Our previous protective association of the A allele of tag SNP 6 (rs10183914) in the PD-Goth discovery study [23] could not be replicated in the meta-analysis including the five replication materials $(+0.3$ year per A allele, $\mathrm{p}=0.23)$.

Exploratory meta-analyses of NFE2L2 individual SNPs, including all six materials, showed no significant associations with risk of PD (Table 5).

Exploratory meta-analyses of NFE2L2 individual SNPs with $\mathrm{AAO}$, including all six materials, showed associations with four SNPs; tag SNP 2 (rs7557529, 1.0 year per A allele, $p=0.042)$, promoter SNP $1(\mathrm{rs} 35652124,1.1$ year per $\mathrm{G}$ allele, $\mathrm{p}=0.045)$, tag SNP $3(\mathrm{rs} 2886161,1.2$ year per $\mathrm{G}$ allele, $\mathrm{p}=0.021)$ and tag SNP $4(\mathrm{rs} 1806649,+1.2$ year per A allele, $\mathrm{p}=0.029$ ) (Table 6).

\section{Discussion}

Since oxidative stress is implicated in the pathogenesis of PD $[2,3]$ and Nrf2 is a central protein in the cellular defense against oxidative stress, genetic variation affecting the efficiency of Nrf2 could contribute to the disease. Here, based on the previous results from our group [23], we performed meta-analyses on five independent patientcontrol materials to investigate the effects of seven haplotypes in the Nrf2-encoding gene NFE2L2 on risk and AAO of PD. In addition, the included SNPs were analyzed individually in exploratory meta-analyses including the PD-Goth discovery material. To our knowledge, with 1038 PD patients and 1600 control subjects included, this is the most extensive attempt yet to investigate the impact of genetic variation in NFE2L2 on PD risk and AAO.

Meta-analyses of three NFE2L2 haplotypes containing five consecutive tag SNPs and three functional promoter SNPs showed association of haplotype GAGCAAAA with both decreased risk and later onset of PD (Tables 3 and 4, Figure 2). This supports our previously observed 
Table 3 Replication of haplotype associations with risk of PD

\begin{tabular}{|c|c|c|c|c|c|c|c|c|c|c|c|c|c|c|c|c|}
\hline \multirow{2}{*}{$\frac{\text { SNP }^{1}}{2,3,4,5,6}$} & \multirow{2}{*}{$\begin{array}{l}\text { Haplotypes } \\
\text { GAAAA }\end{array}$} & \multicolumn{2}{|c|}{$\begin{array}{l}\text { Sweden PD-Goth } \\
\text { OR/allele (CI) }\end{array}$} & \multicolumn{2}{|c|}{$\begin{array}{l}\text { Italy } \\
\text { OR/allele (CI) }\end{array}$} & \multicolumn{2}{|c|}{$\begin{array}{l}\text { Sweden PD-Link } \\
\text { OR/allele (Cl) }\end{array}$} & \multicolumn{2}{|c|}{$\begin{array}{l}\text { Malta } \\
\text { OR/allele (Cl) }\end{array}$} & \multicolumn{2}{|c|}{$\begin{array}{l}\text { Poland } \\
\text { OR/allele (Cl) }\end{array}$} & \multicolumn{2}{|c|}{$\begin{array}{l}\text { Germany } \\
\text { OR/allele (Cl) }\end{array}$} & \multicolumn{2}{|c|}{$\begin{array}{l}\text { Meta-analysis }{ }^{2} \\
\text { OR/allele (CI) }\end{array}$} & \multirow{2}{*}{$\frac{\text { p-value }}{0.052}$} \\
\hline & & $0.8(0.5$ & 1.1) & $1.1(0.9$ & 1.5) & $0.9(0.7$ & 1.2) & $0.8(0.6$ & 1.3) & $0.7(0.5$ & $0.9)$ & $0.7(0.4$ & 1.3) & $0.88(0.76$ & 1.03) & \\
\hline & GAGGG & $2.1(1.2$ & 3.8) & $1.2(0.8$ & 1.7) & $0.9(0.5$ & 1.3) & $0.7(0.4$ & 1.3) & $1.0(0.6$ & 1.6) & $1.1(0.5$ & 2.5) & $1.00(0.81$ & 1.24) & 0.50 \\
\hline & GAAAG & $2.8(1.0$ & 7.7) & $2.8(1.1$ & 6.8) & $0.4(0.1$ & 1.2) & $0.0(0.0$ & $2.910^{18}$ ) & $0.5(0.2$ & 1.7) & $0.0(0.0$ & 21.6) & $0.98(0.54$ & 1.77) & 0.53 \\
\hline P1, P2, P3 & AGC & $1.0(0.7$ & 1.3) & $1.1(0.9$ & 1.3) & $0.8(0.6$ & 1.1) & $1.0(0.7$ & 1.3) & $0.8(0.6$ & 1.0) & $0.9(0.5$ & 1.5) & $0.92(0.81$ & 1.04) & 0.09 \\
\hline \multirow[t]{3}{*}{$2, \mathrm{P1}, \mathrm{P2}, \mathrm{P3}, 3,4,5,6$} & GAGCAAAA & $0.8(0.6$ & 1.1) & $1.1(0.9$ & 1.5) & $0.9(0.7$ & 1.2) & $0.8(0.6$ & 1.3) & $0.5(0.3$ & $0.7)$ & $0.7(0.4$ & 1.3) & $0.84(0.72$ & $0.98)$ & 0.012 \\
\hline & GAGAAGGG & $2.4(1.3$ & 4.5) & $1.2(0.9$ & 1.8) & $0.9(0.6$ & 1.4) & $0.8(0.4$ & 1.4) & $0.6(0.3$ & 1.0) & $1.1(0.5$ & $2.5)$ & $0.95(0.76$ & 1.18) & 0.68 \\
\hline & GAGCAAAG & $2.9(1.0$ & 8.4) & $2.8(1.1$ & 6.8) & $0.4(0.1$ & 1.2) & $0.0(0.0$ & $\left.3.310^{36}\right)$ & $0.4(0.1$ & $1.5)$ & $0.0(0.0$ & 29.2) & $0.95(0.52$ & 1.72) & 0.57 \\
\hline
\end{tabular}

Odds ratios (OR) are presented as absolute numbers $(95 \% \mathrm{Cl})$. Sex was used as covariate for all materials except for Poland, where the number of males and females are identical in patients and controls. Statistically significant $\mathrm{p}$-values are highlighted in bold.

${ }^{1}$ For SNP locations see Figure 1 and Table 2.

${ }^{2}$ The meta-analysis was calculated using inverse-variance weighting of effects with one-sided p-values and included all materials except Sweden PD-Goth, since it is the discovery material on which the haplotype selection was based. 


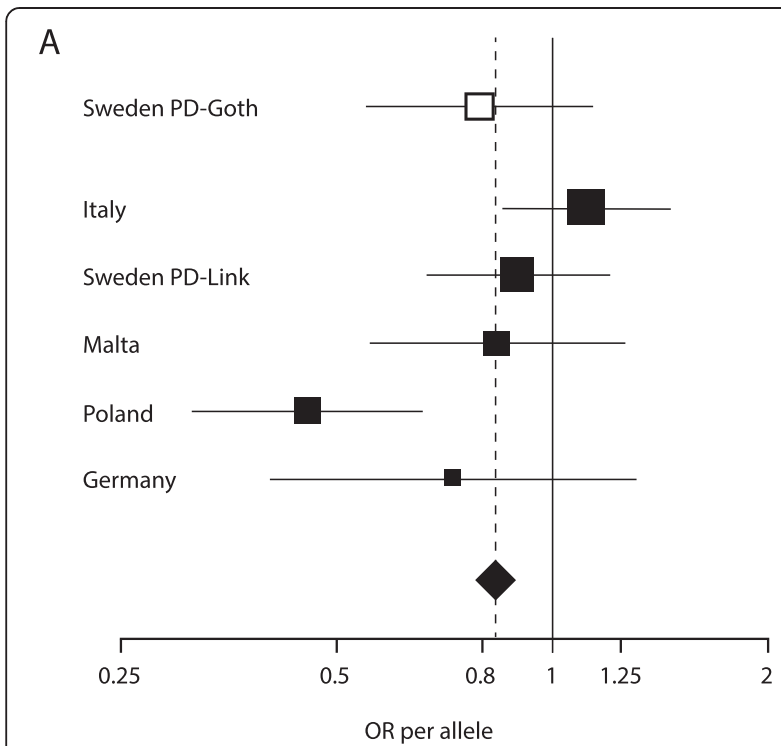

B

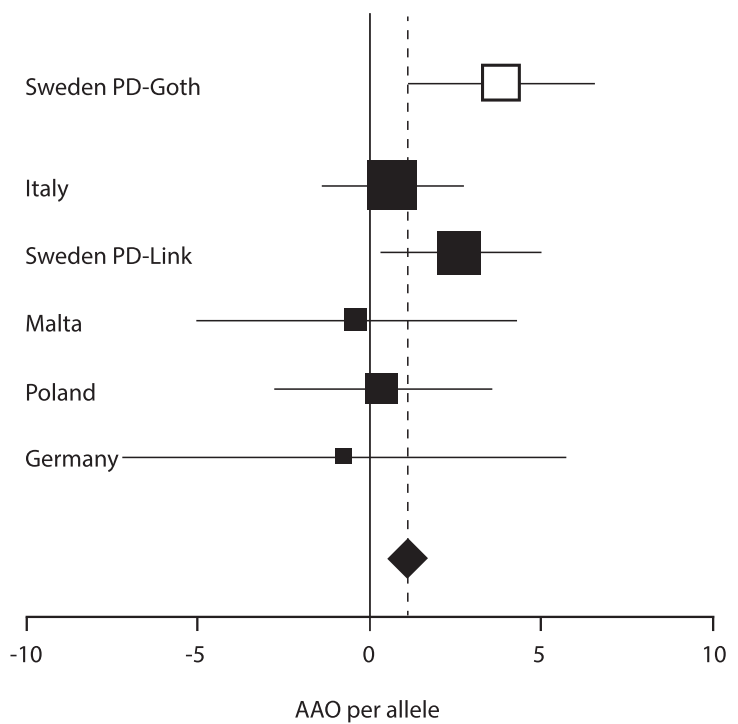

Figure 2 Forest plot showing the NFE2L2 haplotype GAGCAAAA associations with decreased risk (A) and later onset (B) of PD in meta-analysis including the five replication materials: Italy, Sweden PD-Link, Malta, Poland and Germany (filled squares). The previous finding from the Swedish PD-Goth discovery study is also shown (empty squares), though the material was not included in the meta-analysis. The areas of the squares are proportional to the meta-analysis weights (inverse variance).

protective effect of this haplotype, which was associated with later onset of PD in the PD-Goth discovery material and decreased risk of PD in the Polish replication material in the first study [23]. In addition, meta-analyses of the three previously associated NFE2L2 haplotypes in the window including the five tag SNPs only (tag SNPs 2 6) showed association of haplotype GAAAA with later onset of PD. This haplotype also had a tendency towards association with decreased risk of
PD. Obviously, GAGCAAAA and GAAAA are highly influenced by one another and should not be considered separate haplotypes. The promoter haplotype AGC is in LD with the PD-associated haplotype GAAAA $\left(\mathrm{r}^{2}=0.4\right)$ [23] and the PD-associated combined haplotype GAGCAAAA contains the fully functional promoter haplotype AGC $[24,25]$. This suggests that the associations could be driven by genetic variation in the NFE2L2 promoter. However, the fact that the promoter haplotype AGC alone showed no association with PD in metaanalysis indicates that other parts of the gene are involved as well. This is also in accordance with a previous study on a Taiwanese material, where none of the NFE2L2 promoter haplotypes were associated with PD [35].

Haplotypes GAGAAGGG, GAGGG, GAGCAAAG and GAAAG in the same two haplotype windows were all associated with increased risk of PD in the PD-Goth discovery material, but none of the associations could be replicated here. Likewise, the association of tag SNP 6 (rs10183914) with later PD onset in the PD-Goth discovery study could not be replicated in meta-analysis.

In line with our previous results, individual exploratory meta-analyses of the genotyped NFE2L2 SNPs on patient and control subjects from six independent European centers showed no associations with risk of PD. However, in this study four NFE2L2 SNPs were associated with AAO of PD in the exploratory meta-analyses. The minor alleles of tag SNP 2 (rs7557529), promoter SNP P1 (rs35652124) and tag SNP 3 (rs2886161) were all associated with one year earlier PD onset per allele. Tag SNP 3 of our study was previously included in a multiple candidate gene study, but was not significantly associated with PD [36]. Tag SNP 2 is in LD $\left(r^{2}=0.9\right)$ with SNP rs6726395 [23], which was previously found to increase the risk of PD in a first-tier, sib-pair whole-genome study of PD, though the association was not replicated in the second-tier case control study [37]. The minor G allele of promoter SNP P1 has been shown to significantly decrease Nrf2 protein levels [24,25]. Tag SNP 2 and especially tag SNP 3 of our study are both in LD with the minor G allele of P1 $\left(r^{2}=0.4\right.$ and $r^{2}=0.9$, respectively) (Figure 1 ), which could possibly explain the observed associations of these SNPs with earlier PD onset in our study. The minor A allele of tag SNP 4 (rs1806649) was instead protective and delayed onset of PD with one year per allele in meta-analysis. Consistent with the discussion above, this SNP was not in LD with SNP P1 $\left(r^{2}=0.1\right)$. Activation of Nrf2 with natural or synthetic substances have been shown to reduce oxidative stress and neurodegeneration in different PD models $[10,11]$, which suggests that Nrf2 could be a suitable target for pharmacological intervention of PD. In this context, the associations of NFE2L2 variants with AAO of PD are interesting. 
Table 4 Replication of haplotype associations with AAO of PD

\begin{tabular}{|c|c|c|c|c|c|c|c|c|c|c|c|c|c|c|c|}
\hline \multirow{2}{*}{$\frac{\text { SNP }^{1}}{2,3,4,5,6}$} & \multirow{2}{*}{$\begin{array}{l}\text { Haplotypes } \\
\text { GAAAA }\end{array}$} & \multicolumn{2}{|c|}{$\begin{array}{l}\text { Sweden PD-Goth } \\
\text { Years/allele (Cl) }\end{array}$} & \multicolumn{2}{|c|}{$\begin{array}{l}\text { Italy } \\
\text { Years/allele (Cl) }\end{array}$} & \multicolumn{2}{|c|}{$\begin{array}{l}\text { Sweden PD-Link } \\
\text { Years/allele (CI) }\end{array}$} & \multicolumn{2}{|l|}{$\begin{array}{l}\text { Malta } \\
\text { Years/allele (Cl) }\end{array}$} & \multicolumn{2}{|c|}{$\begin{array}{l}\text { Poland } \\
\text { Years/allele (Cl) }\end{array}$} & $\begin{array}{l}\text { Germany } \\
\text { Years/allele (Cl) }\end{array}$ & \multicolumn{2}{|c|}{$\begin{array}{l}\text { Meta-analysis }^{2} \\
\text { Years/allele (Cl) }\end{array}$} & \multirow{2}{*}{$\frac{p \text {-value }}{0.024}$} \\
\hline & & $+4.1(+1.3$ & $+6.8)$ & $+0.6(-1.5$ & $+2.6)$ & $+2.8(+0.5$ & $+5.1)$ & $-0.4(-5.0+4.3$ & & $+1.2(-1.6$ & $+4.0)$ & $-0.2\left(\begin{array}{ll}-6.8 & +6.5\end{array}\right)$ & $+1.28(-0.01$ & $+2.54)$ & \\
\hline & GAGGG & $-0.9(-4.6$ & -32.8) & $0.4(-2.9$ & $+2.2)$ & $+2.1(-1.6$ & $+6.0)$ & $+0.5(-7.0+$ & & 2 & -2.8) & $+1.3(-5.4 \quad+8.0)$ & 50 & 39) & 0.59 \\
\hline & GAAAG & $-3.6(-9.1$ & $+2.0)$ & $-0.2(-5.7$ & $+5.4)$ & $+1.0(-8.7$ & $+10.7)$ & $+253.1(-483.1$ & 9.3) & $-1.2(-10.8$ & $+8.4)$ & $-60.9(-221.2+99.3)$ & $-0.16(-4.46$ & $+4.14)$ & 0.47 \\
\hline I, P2, P3 & AGC & $+1.4(-0.7$ & $+3.6)$ & $+0.8(-0.8$ & $+2.4)$ & $-0.1(-2.1$ & $+1.9)$ & $+0.3(-3.1+3.7$ & & $-0.0(-2.2$ & $+2.1)$ & $-1.6(-6.2+2.9)$ & $+0.22(-0.79$ & $+1.23)$ & 0.3 \\
\hline \multirow{3}{*}{$\begin{array}{l}2, \mathrm{P} 1, \mathrm{P} 2, \mathrm{P} 3, \\
3,4,5,6\end{array}$} & AG & $+3.8(+1.1$ & $+6.5)$ & $+0.7(-1.4$ & $+2.7)$ & $+2.6(+0.3$ & $+5.0)$ & $-0.4(-5.0+$ & & $+0.4(-2.8$ & $+3.6)$ & $-0.7(-7.2 \quad+5.7)$ & +1 & $+2.40)$ & 0.048 \\
\hline & GAGAAGGG & $-0.8(-4.6$ & $+2.9)$ & $-0.3(-2.9$ & $+2.2)$ & $+2.1(-1.7$ & $+6.0)$ & $-0.5(-7.0+8.0$ & & $+0.5(-3.7$ & $+4.8)$ & $+1.3(-5.4 \quad+8.0)$ & $+0.51(-1.26$ & $+2.28)$ & 0.71 \\
\hline & GAGCAAAG & $-3.0(-8.6$ & $+2.6)$ & $+0.2(-5.4$ & $+5.8)$ & $+0.8(-9.1$ & $+10.6)$ & $-2817.1(-5873.3$ & $+239.1)$ & $-0.6(-11.4$ & $+10.2)$ & $-134.6(-321.2+51.9)$ & $+0.09(-4.35$ & $+4.52)$ & 0.52 \\
\hline
\end{tabular}

Effects on AAO are given as absolute numbers $(95 \% \mathrm{Cl})$. Sex was used as covariate for all materials. Statistically significant $\mathrm{p}$-values are highlighted in bold.

${ }^{1}$ For SNP locations see Figure 1 and Table 2.

${ }^{2}$ The meta-analysis was calculated using inverse-variance weighting of effects with one-sided p-values and included all materials except Sweden PD-Goth, since it is the discovery material on which the haplotype selection was based. 
Table 5 Individual SNP associations with risk of PD

\begin{tabular}{|c|c|c|c|c|c|c|c|c|c|c|c|c|c|c|c|c|c|}
\hline \multicolumn{2}{|c|}{$\mathrm{SNP}^{1}$} & \multirow{2}{*}{$\begin{array}{l}\text { Genotype } \\
G>A\end{array}$} & \multicolumn{2}{|c|}{$\begin{array}{l}\text { Sweden PD-Goth } \\
\text { OR/allele (Cl) }\end{array}$} & \multicolumn{2}{|c|}{$\begin{array}{l}\text { Italy } \\
\text { OR/allele (CI) }\end{array}$} & \multicolumn{2}{|c|}{$\begin{array}{l}\text { Sweden PD-Link } \\
\text { OR/allele (CI) }\end{array}$} & \multicolumn{2}{|c|}{$\begin{array}{l}\text { Malta } \\
\text { OR/allele (CI) }\end{array}$} & \multicolumn{2}{|c|}{$\begin{array}{l}\text { Poland } \\
\text { OR/allele (CI) }\end{array}$} & \multicolumn{2}{|c|}{$\begin{array}{l}\text { Germany } \\
\text { OR/allele (CI) }\end{array}$} & \multicolumn{2}{|c|}{$\begin{array}{l}\text { Meta-analysis }{ }^{2} \\
\text { OR/allele (Cl) }\end{array}$} & \multirow{2}{*}{$\frac{\text { p-value }}{0.18}$} \\
\hline 2 & rs7557529 & & $0.9(0.7$ & 1.2) & $0.8(0.7$ & 1.0) & $1.2(1.0$ & 1.6) & $1.5(1.1$ & 2.0) & $1.3(1.0$ & 1.8) & $1.0(0.6$ & 1.7) & $1.08(0.96$ & 1.21) & \\
\hline & rs35652124 & $A>G$ & $0.8(0.6$ & 1.2) & $0.7(0.6$ & $0.9)$ & $1.3(1.0$ & 1.7) & $0.9(0.6$ & 1.3) & $1.3(1.0$ & 1.8) & $1.1(0.6$ & 1.8) & $0.99(0.87$ & 1.12) & 0.86 \\
\hline P2 & rs6706649 & $G>A$ & $0.9(0.6$ & 1.4) & $1.3(0.9$ & $1.8)$ & $1.1(0.7$ & 1.5) & $1.6(1.0$ & 2.4) & $1.0(0.7$ & $1.5)$ & $0.9(0.4$ & 2.0) & $1.14(0.96$ & 1.35) & 0.14 \\
\hline P3 & rs6721961 & $C>A$ & $1.8(1.1$ & 3.0) & $1.1(0.8$ & $1.5)$ & $0.9(0.6$ & 1.4) & $0.7(0.4$ & 1.1) & $1.0(0.7$ & 1.6) & $1.3(0.7$ & 2.6) & $1.07(0.90$ & 1.27) & 0.45 \\
\hline 3 & rs2886161 & $A>G$ & $0.8(0.6$ & 1.2) & $0.7(0.5$ & $0.9)$ & $1.3(1.0$ & 1.7) & $1.0(0.7$ & 1.4) & $1.3(1.0$ & $1.8)$ & $1.1(0.6$ & 1.8) & $0.98(0.87$ & 1.12) & 0.81 \\
\hline 4 & rs1806649 & $G>A$ & $1.0(0.7$ & 1.3) & $1.2(0.9$ & $1.5)$ & $0.8(0.6$ & 1.1) & $0.8(0.6$ & $1.2)$ & $0.7(0.5$ & $0.9)$ & $0.7(0.4$ & $1.2)$ & $0.88(0.77$ & 1.01) & 0.07 \\
\hline 5 & rs2001350 & $A>G$ & $2.0(1.1$ & 3.6) & $1.3(1.0$ & $1.8)$ & $0.9(0.6$ & 1.3) & $0.8(0.4$ & 1.4) & $0.9(0.6$ & 1.4) & $1.0(0.5$ & 2.1) & $1.10(0.91$ & 1.34) & 0.31 \\
\hline 6 & 10183914 & $G>A$ & $0.8(0.6$ & 1.2) & $1.1(0.9$ & 1.4) & $0.8(0.6$ & 1.1) & $1.0(0.7$ & 1.4) & $0.8(0.6$ & 1.1) & $0.9(0.6$ & 1.6) & $0.93(0.82$ & 1.05) & 0.22 \\
\hline
\end{tabular}

Odds ratios (OR) are given for the minor allele and presented as absolute numbers $(95 \% \mathrm{Cl})$. Sex was used as covariate for all materials except for Poland, where the number of males and females are identical in patients and controls.

and Table 2.

${ }^{2}$ The meta-analysis was calculated using inverse-variance weighting of effects with two-sided p-values, including all six materials. 
Table 6 Individual SNP associations with AAO of PD

\begin{tabular}{|c|c|c|c|c|c|c|c|c|c|c|c|c|c|c|c|c|c|}
\hline \multicolumn{2}{|c|}{$\mathrm{SNP}^{1}$} & \multirow{2}{*}{$\begin{array}{l}\text { Genotype } \\
G>A\end{array}$} & \multicolumn{2}{|c|}{$\begin{array}{l}\text { Sweden PD-Goth } \\
\text { Years/allele (Cl) }\end{array}$} & \multicolumn{2}{|c|}{$\begin{array}{l}\text { Italy } \\
\text { Years/allele (Cl) }\end{array}$} & \multicolumn{2}{|c|}{$\begin{array}{l}\text { Sweden PD-Link } \\
\text { Years/allele (Cl) }\end{array}$} & \multicolumn{2}{|c|}{$\begin{array}{l}\text { Malta } \\
\text { Years/allele (Cl) }\end{array}$} & \multicolumn{2}{|c|}{$\begin{array}{l}\text { Poland } \\
\text { Years/allele (Cl) }\end{array}$} & \multicolumn{2}{|c|}{$\begin{array}{l}\text { Germany } \\
\text { Years/allele (CI) }\end{array}$} & \multicolumn{2}{|c|}{$\begin{array}{l}\text { Meta-analysis }^{2} \\
\text { Years/allele (Cl) }\end{array}$} & \multirow{2}{*}{$\frac{p \text {-value }}{0.042}$} \\
\hline 2 & rs7557529 & & $-1.7(-3.9$ & $+0.6)$ & $-0.4(-2.0$ & $+1.3)$ & $-2.2(-4.3$ & $-0.1)$ & $-0.8(-4.7$ & $+3.1)$ & $-0.2(-2.4$ & $+2.0)$ & $-0.8(-6.0$ & $+4.3)$ & $-0.99(-1.94$ & $-0.03)$ & \\
\hline P1 & rs35652124 & $>\mathrm{G}$ & $-2.1(-4.5$ & $+0.3)$ & $1.3(-3.3$ & $+0.1)$ & $-1.8(-$ & $+0.5)$ & $.6(-5.8$ & $+2.6)$ & $0.2(-2.0$ & $+2.4)$ & $+2.8(-2.3$ & $+7.8)$ & 12 & $-0.02)$ & 045 \\
\hline P2 & rs6706649 & $G>A$ & $+1.4(-1.8$ & $+4.7)$ & $+1.4(-0.9$ & $+3.8)$ & $+1.4(-1.5$ & $+4.2)$ & $-0.0(-5.2$ & $+5.2)$ & $-0.6(-3.7$ & $+2.5)$ & $-1.7(-9.5$ & $+6.0)$ & $+0.85(-0.49$ & $+2.19)$ & 0.22 \\
\hline P3 & rs6721961 & $C>A$ & $-1.5(-5.0$ & $+2.0)$ & $-0.7(-2.7$ & $+1.3)$ & $+2.1(-1.4$ & $+5.6)$ & $+2.7(-3.2$ & $+8.6)$ & $+0.3(-2.8$ & $+3.3)$ & $+0.0(-6.0$ & $+6.1)$ & $-0.81(-2.03$ & $+0.41)$ & 9 \\
\hline 3 & rs2886161 & $A>G$ & $-2.4(-4.8$ & $-0.1)$ & $-1.4(-3.4$ & $+0.6)$ & $-2.0(-4.3$ & $+0.3)$ & $-1.5(-5.6$ & $+2.7)$ & $+0.0(-2.2$ & $+2.2)$ & $+2.7(-2.4$ & $+7.8)$ & $-1.23(-2.27$ & $-0.18)$ & 1 \\
\hline 4 & rs1806649 & $G>A$ & $+2.7(+0.2$ & $+5.1)$ & $+0.4(-1.5$ & $+2.4)$ & $+2.7(+0.4$ & $+4.9)$ & $-2.4(-7.0$ & $+2.1)$ & $+1.0(-1.7$ & $+3.7)$ & $-3.6(-9.5$ & $+2.3)$ & $+1.20(+0.12$ & $+2.28)$ & 0.029 \\
\hline 5 & 350 & $>\mathrm{G}$ & $-0.6(-4.3$ & $+3.0)$ & $-0.8(-3.1$ & $+1.6)$ & $+1.8(-1.9$ & $+5.6)$ & $+1.8(-5.1$ & $+8.8)$ & $-0.7(-4.1$ & $+2.8)$ & $+1.1(-5.7$ & $+7.8)$ & $-0.11(-1.59$ & $+1.37)$ & 0.88 \\
\hline 6 & 10183914 & $G>A$ & $+3.7(+1.3$ & $+6.2)$ & $+0.3(-1.3$ & $+2.0)$ & $+1.5(-0.6$ & $+3.7)$ & $-0.4(-4.2$ & $+3.3)$ & $-0.1(-2.4$ & $+2.3)$ & $-1.4(-6.2$ & $+3.5)$ & $+0.95(-0.04$ & +1.94) & 0.06 \\
\hline
\end{tabular}

Effects on AAO are given for the minor allele and presented as absolute numbers $(95 \% \mathrm{Cl})$. Sex was used as covariate for all materials. Statistically significant p-values are highlighted in bold.

${ }^{1}$ For SNP locations see Figure 1 and Table 2.

${ }^{2}$ The meta-analysis was calculated using inverse-variance weighting of effects with two-sided p-values, including all six materials. 
Though haplotype GAGCAAAA showed a trend towards increased risk of PD in the Italian material when the materials were analyzed individually, this haplotype decreased the risk of PD in meta-analysis (Table 3, Figure 2A). Idiopathic PD is a multifactorial disease caused by several risk genes, possibly in combination with environmental factors. This may explain why the effect of a risk gene could be more obvious in some materials than in others. Also, study design as well as different genetic background in both PD patients and controls could affect the outcome of a multicenter study. Considering this, despite the relatively small effects in the meta-analyses, the replicated associations between genetic variation in the NFE2L2 gene and idiopathic PD are interesting and should be investigated further in functional studies.

\section{Conclusions}

Meta-analyses including five independent patient-control materials from different European centers confirmed protective effects of NFE2L2 haplotypes GAGCAAAA and GAAAA against PD. In addition, exploratory meta-analyses revealed four individual SNPs affecting the AAO of PD, including a functional SNP located in the NFE2L2 promoter. In summary, our data support the hypothesis that variation in the Nrf2-encoding gene NFE2L2 may contribute to the pathogenesis of idiopathic PD.

\section{Additional files}

Additional file 1: Table S1. Individual SNP frequencies in PD patients and control subjects.

Additional file 2: Table S2. Haplotype frequencies in PD patients and control subjects.

\section{Abbreviations \\ AAD: Age at diagnosis; AAFS: Age at first symptom; AAO: Age at onset; AAS: Age at sampling; ARE: Antioxidant response element; DA: Dopamine; DAergic: Dopaminergic; FH: Family history; HO-1: Heme oxygenase 1; iPSCs: Induced pluripotent stem cells; Keap1: Kelch-like ECH-associated protein 1; LD: Linkage disequilibrium; MPTP: 1-methyl-4-phenyl-1,2,3, 6-tetrahydropyridine; NFE2L2: NF-E2-like 2; Nrf2: Nuclear factor erythroid 2 (NF-E2) related factor 2; NQO1: NAD(P)H dehydrogenase, quinone 1; PD: Parkinson s disease; SNP: Single nucleotide polymorphism; SN: Substantia nigra.}

\section{Competing interests}

The authors declare that they have no competing interests.

\section{Authors contributions}

MVO: Research project Organization, design; Laboratory work Execution; Statistical analysis Design and execution; Manuscript Review and critique. PB: Statistical analysis Execution; Manuscript Writing. AQ: Laboratory work Supplier of patient material; Manuscript Review and critique. VDM: Laboratory work Supplier of patient material; Manuscript Review and critique. GA: Laboratory work Supplier of patient material; Manuscript Review and critique. PS: Laboratory work Supplier of patient material; Manuscript Review and critique. SBW: Laboratory work Supplier of patient material; Manuscript Review and critique. MD: Laboratory work Supplier of patient material; Manuscript Review and critique. MB: Laboratory work Supplier of patient material; Manuscript Review and critique. HN: Laboratory work Supplier of patient material; Manuscript Review and critique. CK: Laboratory work Supplier of patient material; Manuscript Review and critique. MN: Research project Conception; Manuscript Review and critique. OH: Research project Conception; Manuscript Review and critique. SN: Statistical analysis Design, review and critique; Manuscript Review and critique. HZ: Research project Conception; Manuscript Review and critique. All authors have read and approved the final manuscript.

\section{Acknowledgements}

We are indebted to the patients who participated in this study. The authors are grateful for the excellent technical assistance of Mrs. Mona Seibt Palmr. This work was supported by grants from the Swedish Research Council, the Knut and Alice Wallenberg Foundation, the Sahlgrenska University Hospital, the West Sweden RUN fundings, Edith Jacobsson Foundation, Axel Linder s Foundation, the Gteborg Medical Society, the Swedish Medical Society, Swedish Brain Power, Stiftelsen fr Gamla Tjnarinnor, the Swedish Parkinson Foundation, the Foundation for Parkinson Research at Linkping University (Stiftelsen fr Parkinsonforskning), Sweden, Gun and Bertil Stohne s

Foundation, the hln Foundation, the Alzheimer s Foundation, Sweden, the Assar Gabrielsson Foundation, The Swedish Cancer Foundation, The Swedish Pain Foundation and the Herman and Lilly Schilling Foundation. The samples from Malta were from the Malta BioBank, a founding partner of the Eurobiobank. They were collected by the Laboratory of Molecular Genetics, University of Malta, as part of the Geoparkinson Study funded by the Fifth Framework programme of the European Commission, project number QLK4CT-1999-01133. Funding sources played no role in the design and conduct of the study; collection, management, analysis, and interpretation of the data; or preparation, review, or approval of the manuscript.

\section{Author details}

${ }^{1}$ Institute of Neuroscience and Physiology, Department of Psychiatry and Neurochemistry, The Sahlgrenska Academy at the University of Gothenburg, Bl strket 15, 41345 Gothenburg, Sweden. IInstitute of Neurology, University Magna Graecia, Catanzaro, Italy. ${ }^{3}$ Neuroimaging Research Unit, Institute of Molecular Bioimaging and Physiology, National Research Council, Catanzaro, Italy. ${ }^{4}$ Institute of Neurological Sciences, National Research Council, Cosenza, Italy. ${ }^{5}$ Institute of Molecular Bioimaging and Physiology, Section of Germaneto, National Research Council, Catanzaro, Italy. ${ }^{6}$ Division of Cell Biology, Department of Clinical and Experimental Medicine, Linkping University, SE-581 85 Linkping, Sweden. ${ }^{7}$ Department of Applied Biomedical Science, Faculty of Health Sciences, University of Malta, Msida, Malta. ${ }^{8}$ Department of Pharmacology, Pomeranian Medical University, Powstancow Wlkp. 72, Szczecin 70-111, Poland. ${ }^{9}$ Institute of Neuroscience and Physiology, Department of Pharmacology, the Sahlgrenska Academy at the University of Gothenburg, Box 43140530 Gothenburg, Sweden. ${ }^{10}$ Institute of Neurogenetics, University of Luebeck, Luebeck, Germany. ${ }^{11}$ Institute of Neuroscience and Physiology, Center for Brain Repair and Rehabilitation, the Sahlgrenska Academy at the University of Gothenburg, Per Dubbsgatan 14, 41345 Gothenburg, Sweden. ${ }^{12}$ Hunter Medical Research Institute, University of Newcastle, Newcastle, Australia. ${ }^{13}$ Institute of Biomedicine, Department of Clinical Chemistry and Transfusion Medicine, The Sahlgrenska Academy at the University of Gothenburg, Bruna Strket 16, 41345 Gothenburg, Sweden. ${ }^{14}$ Institute of Mathematical Sciences, Department of Mathematical Statistics, Chalmers University of Technology, Chalmers tvrgata 3, 41296 Gothenburg, Sweden. ${ }^{15} \mathrm{UCL}$ Institute of Neurology, Queen Square, London WC1N 3BG, UK.

Received: 26 August 2014 Accepted: 1 December 2014 Published online: 12 December 2014

\section{References}

1. Lees AJ, Hardy J, Revesz T: Parkinsons disease. Lancet 2009, 373(9680):2055 2066.

2. Zhou C, Huang Y, Przedborski S: Oxidative stress in Parkinson s disease: a mechanism of pathogenic and therapeutic significance. Ann N Y Acad Sci 2008, 1147:93 104.

3. Chinta SJ, Andersen JK: Redox imbalance in Parkinson s disease. Biochim Biophys Acta 2008, 1780(11):1362 1367.

4. Copple IM, Goldring CE, Kitteringham NR, Park BK: The Nrf2-Keap1 defence pathway: role in protection against drug-induced toxicity. Toxicology 2008, 246(1):24 33 . 
5. Itoh K, Wakabayashi N, Katoh Y, Ishii T, Igarashi K, Engel JD, Yamamoto M: Keap1 represses nuclear activation of antioxidant responsive elements by Nrf2 through binding to the amino-terminal Neh2 domain. Genes Dev 1999, 13(1):76 86.

6. Cullinan SB, Gordan JD, Jin J, Harper JW, Diehl JA: The Keap1-BTB protein is an adaptor that bridges Nrf2 to a Cul3-based E3 ligase: oxidative stress sensing by a Cul3-Keap1 ligase. Mol Cell Biol 2004, 24(19):8477 8486.

7. Furukawa $\mathrm{M}$, Xiong $\mathrm{Y}$ : BTB protein Keap1 targets antioxidant transcription factor Nrf2 for ubiquitination by the Cullin 3-Roc1 ligase. Mol Cell Biol 2005, 25(1):162 171.

8. Itoh K, Chiba T, Takahashi S, Ishii T, Igarashi K, Katoh Y, Oyake T, Hayashi N, Satoh K, Hatayama I, Yamamoto M, Nabeshima Y: An Nrf2/small Maf heterodimer mediates the induction of phase II detoxifying enzyme genes through antioxidant response elements. Biochem Biophys Res Commun 1997, 236(2):313 322

9. Magesh S, Chen Y, Hu L: Small molecule modulators of Keap1-Nrf2-ARE pathway as potential preventive and therapeutic agents. Med Res Rev 2012, 32(4):687 726.

10. Kumar H, Koppula S, Kim IS, More SV, Kim BW, Choi DK: Nuclear factor erythroid 2-related factor 2 signaling in Parkinson disease: a promising multi therapeutic target against oxidative stress, neuroinflammation and cell death. CNS \& neurological disorders drug targets 2012, 11(8):1015 1029.

11. Yang $Y$, Jiang $S$, Yan J, Li Y, Xin Z, Lin Y, Qu Y: An overview of the molecular mechanisms and novel roles of Nrf2 in neurodegenerative disorders. Cytokine Growth Factor Rev 2014, Epub ahead of print.

12. Cook AL, Vitale AM, Ravishankar S, Matigian N, Sutherland GT, Shan J, Sutharsan R, Perry C, Silburn PA, Mellick GD, Whitelaw ML, Wells CA, Mackay-Sim A, Wood SA: NRF2 activation restores disease related metabolic deficiencies in olfactory neurosphere-derived cells from patients with sporadic Parkinson s disease. PLoS ONE 2011, 6(7):e21907.

13. Meesarapee B, Thampithak A, Jaisin $Y$, Sanvarinda P, Suksamrarn A, Tuchinda $P$, Morales NP, Sanvarinda Y: Curcumin I mediates neuroprotective effect through attenuation of quinoprotein formation, p-p38 MAPK expression, and caspase-3 activation in 6-hydroxydopamine treated SH-SY5Y cells. Phytother Res 2014, 28(4):611 616.

14. Kaidery NA, Banerjee R, Yang L, Smirnova NA, Hushpulian DM, Liby KT, Williams CR, Yamamoto M, Kensler TW, Ratan RR, Sporn MB, Beal MF, Gazaryan IG, Thomas B: Targeting Nrf2-mediated gene transcription by extremely potent synthetic triterpenoids attenuate dopaminergic neurotoxicity in the MPTP mouse model of Parkinson s disease. Antioxidants \& redox signaling 2013, 18(2):139 157.

15. Wild AC, Moinova HR, Mulcahy RT: Regulation of gamma-glutamylcysteine synthetase subunit gene expression by the transcription factor Nrf2. The Journal of biological chemistry 1999, 274(47):33627 33636.

16. Alam J, Stewart D, Touchard C, Boinapally S, Choi AM, Cook JL: Nrf2, a Cap n Collar transcription factor, regulates induction of the heme oxygenase-1 gene. The Journal of biological chemistry 1999 274(37):26071 26078

17. Chen K, Gunter K, Maines MD: Neurons overexpressing heme oxygenase-1 resist oxidative stress-mediated cell death. J Neurochem 2000, 75(1):304 313.

18. Venugopal R, Jaiswal AK: Nrf1 and Nrf2 positively and c-Fos and Fra1 negatively regulate the human antioxidant response element-mediated expression of $\mathrm{NAD}(\mathrm{P}) \mathrm{H}$ :quinone oxidoreductase1 gene. Proc Natl Acad Sci USA 1996, 93(25):14960 14965

19. Segura-Aguilar J, Lind C: On the mechanism of the Mn3(+)-induced neurotoxicity of dopamine:prevention of quinone-derived oxygen toxicity by DT diaphorase and superoxide dismutase. Chem Biol Interact 1989, 72(3):309 324

20. van Muiswinkel FL, de Vos RA, Bol JG, Andringa G, Jansen Steur EN, Ross D, Siegel D, Drukarch B: Expression of NAD(P)H:quinone oxidoreductase in the normal and Parkinsonian substantia nigra. Neurobiol Aging 2004 25(9):1253 1262

21. Ramsey CP, Glass CA, Montgomery MB, Lindl KA, Ritson GP, Chia LA Hamilton RL, Chu CT, Jordan-Sciutto KL: Expression of Nrf2 in neurodegenerative diseases. J Neuropathol Exp Neurol 2007, 66(1):75 85.

22. Imaizumi Y, Okada Y, Akamatsu W, Koike M, Kuzumaki N, Hayakawa H, Nihira T, Kobayashi T, Ohyama M, Sato S, Takanashi M, Funayama M, Hirayama A, Soga T, Hishiki T, Suematsu M, Yagi T, Ito D, Kosakai A, Hayashi K, Shouji M, Nakanishi A, Suzuki N, Mizuno Y, Mizushima N, Amagai M, Uchiyama Y, Mochizuki $\mathrm{H}$, Hattori N, Okano H: Mitochondrial dysfunction associated with increased oxidative stress and alpha-synuclein accumulation in PARK2 iPSC-derived neurons and postmortem brain tissue. Mol Brain 2012, 5:35

23. von Otter M, Landgren S, Nilsson S, Celojevic D, Bergstrom P, Hakansson A Nissbrandt H, Drozdzik M, Bialecka M, Kurzawski M, Blennow K, Nilsson M, Hammarsten O, Zetterberg $\mathrm{H}$ : Association of Nrf2-encoding NFE2L2 haplotypes with Parkinson s disease. BMC Med Genet 2010, 11:36.

24. Marczak ED, Marzec J, Zeldin DC, Kleeberger SR, Brown NJ, Pretorius M Lee CR: Polymorphisms in the transcription factor NRF2 and forearm vasodilator responses in humans. Pharmacogenet Genomics 2012, 22(8):620 628

25. Marzec JM, Christie JD, Reddy SP, Jedlicka AE, Vuong H, Lanken PN, Aplenc R, Yamamoto T, Yamamoto M, Cho HY, Kleeberger SR: Functional polymorphisms in the transcription factor NRF2 in humans increase the risk of acute lung injury. Faseb J 2007, 21(9):2237 2246.

26. Bergman O, Hakansson A, Westberg L, Nordenstrom K, Carmine Belin A, Sydow O, Olson L, Holmberg B, Eriksson E, Nissbrandt H: PITX3 polymorphism is associated with early onset Parkinsons disease. Neurobiol Aging 2010, 31(1):114 117.

27. Westerlund M, Belin AC, Anvret A, Hakansson A, Nissbrandt H, Lind C, Sydow O, Olson L, Galter D: Cerebellar alpha-synuclein levels are decreased in Parkinson s disease and do not correlate with SNCA polymorphisms associated with disease in a Swedish material. Faseb J 2008, 22(10):3509 3514.

28. Dick FD, De Palma G, Ahmadi A, Scott NW, Prescott GJ, Bennett J, Semple S, Dick S, Counsell C, Mozzoni P, Haites N, Wettinger SB, Mutti A, Otelea M, Seaton A, Sderkvist P, Felice A, Geoparkinson study group: Environmental risk factors for Parkinson s disease and parkinsonism: the Geoparkinson study. Occup Environ Med 2007, 64(10):666 672.

29. International HapMap Consortium: The international HapMap project. Nature 2003, 426(6968):789 796.

30. Barrett JC, Fry B, Maller J, Daly MJ: Haploview: analysis and visualization of LD and haplotype maps. Bioinformatics (Oxford, England) 2005, 21(2):263 265

31. Gabriel SB, Schaffner SF, Nguyen H, Moore JM, Roy J, Blumenstiel B, Higgins J DeFelice M, Lochner A, Faggart M, Liu-Cordero SN, Rotimi C, Adeyemo A Cooper R, Ward R, Lander ES, Daly MJ, Altshuler D: The structure of haplotype blocks in the human genome. Science 2002, 296(5576):2225 2229.

32. Livak KJ: Allelic discrimination using fluorogenic probes and the $5^{\prime}$ nuclease assay. Genet Anal 1999, 14(5 6):143 149.

33. Altman DG: Practical statistics for medical research. New York: Chapman and Hall; 1991.

34. Excoffier L, Slatkin M: Maximum-likelihood estimation of molecular haplotype frequencies in a diploid population. Mol Biol Evol 1995, 12(5):921 927

35. Chen YC, Wu YR, Wu YC, Lee-Chen GJ, Chen CM: Genetic analysis of NFE2L2 promoter variation in Taiwanese Parkinson s disease. Parkinsonism Relat Disord 2013, 19(2):247 250.

36. Mizuta I, Satake W, Nakabayashi Y, Ito C, Suzuki S, Momose Y, Nagai Y, Oka A Inoko H, Fukae J, Saito Y, Sawabe M, Murayama S, Yamamoto M, Hattori N, Murata M, Toda T: Multiple candidate gene analysis identifies alpha-synuclein as a susceptibility gene for sporadic Parkinsons disease. Hum Mol Genet 2006 15(7):1151 1158

37. Maraganore DM, de Andrade M, Lesnick TG, Strain KJ, Farrer MJ, Rocca WA Pant PV, Frazer KA, Cox DR, Ballinger DG: High-resolution whole-genome association study of Parkinson disease. Am J Hum Genet 2005, 77(5):685 693

doi:10.1186/s12881-014-0131-4

Cite this article as: von Otter et al:: Genetic associations of Nrf2-encoding NFE2L2 variants with Parkinson s disease a multicenter study. $B M C$ Medical Genetics 2014 15:131. 